EPJ Web of Conferences 41, 08016 (2013)

DOI: $10.1051 /$ epjconf/20134108016

(C) Owned by the authors, published by EDP Sciences, 2013

\title{
Time Domain Characterization of Light Trapping States in Thin Film Solar Cells
}

\author{
M. Birlo ${ }^{1}$, D. Differt ${ }^{1}$, F. Lükermann ${ }^{1}$, W. Pfeiffer $^{1}$, and H. Stiebig ${ }^{1,2}$ \\ ${ }^{1}$ Fakultät für Physik, Universität Bielefeld, 33615 Bielefeld, Germany \\ ${ }^{2}$ Malibu GmbH \& Co. KG, Böttcherstr. 7, 33609 Bielefeld, Germany \\ E-Mail: pfeiffer@physik.uni-bielefeld.de
}

\begin{abstract}
Spectral interferometry of the backscattered radiation reveals coherence lifetimes of about 150 fs for nanolocalized electromagnetic modes in textured layered nanostructures as they are commonly used in thin film photovoltaics to achieve high cell efficiencies.
\end{abstract}

\section{Introduction}

Nanolocalization of light is not only relevant for achieving high spatial resolution in spectroscopy and microscopy but also substantially affects the efficiency of light matter interaction. For example, in thin film solar cells light trapping, i.e. the localization of light in the active layer, determines critically the efficiency [1-3]. It has been shown that randomly textured interfaces increase the light trapping efficiency. Thus, for example, silicon thin film solar cells usually have corrugated internal interfaces between the transparent conductive oxide (TCO, in our case $\mathrm{ZnO}$ ) layers and the absorber medium, i.e. hydrogenated amorphous $\mathrm{Si}(\mathrm{a}-\mathrm{Si}: \mathrm{H})$. Light localization in random stratified media has attracted substantial attention [4-6], however, it is still an unresolved question what type of interface corrugation leads to the best light trapping.

Multiple scattering at corrugated interfaces supports the formation of weakly localized photonic modes similar to Anderson localization of light in three dimensional turbid media [7]. In contrast to diffusive transport these modes appear as resonances and exhibit lifetimes of about $150 \mathrm{fs}$. Here we report the time domain characterization of scattered coherent radiation from layered nanostructures. It is shown that spectral interferometry is applicable to investigate light trapping, allows quantifying trapping state lifetimes, and could serve as a new characterization tool for thin film processing.

\section{Methods and Results}

A modified Mach-Zehnder interferometer is used to measure spectral interferometry for two independent polarization components of the diffuse backscattered light from the sample mounted in one interferometer arm (Fig. 1a). Linearly polarized laser pulses from a Ti:Sapphire oscillator $(795 \mathrm{~nm}, 20 \mathrm{fs}$ ) are focused onto the nanostructure (about $5 \mu \mathrm{m}$ spot diameter). The sample surface is tilted by $10^{\circ}$ with respect to the incident beam. The parabolic mirror recollimates part of the scattered light that is back-reflected under an angle of about $10^{\circ}$ with respect to surface normal. This scattered light is brought to interference with a bandwidth-limited attenuated reference pulse

This is an Open Access article distributed under the terms of the Creative Commons Attribution License 2.0, which permits unrestricted use, distribution, and reproduction in any medium, provided the original work is properly cited. 
propagating in the other interferometer arm that is linearly polarized at $45^{\circ}$ with respect to the interferometer plane. A fibre coupled dual channel spectrograph is used to measure simultaneously the spectral interference for both polarization components. The spectral phase of the reference pulse is characterized using a SPIDER setup.

The layered nanostructures studied here are shown in Fig. 1b,c. The amorphous silicon layer is either grown on a corrugated $\mathrm{ZnO}$ layer (Fig. 1b and left cross section in Fig. 1c) or on a smooth layer (right cross section in Fig. 1c). The corrugation of the initial $\mathrm{ZnO}$ layer is conserved during the growth of the thin silicon layer and thus both interfaces exhibit an almost identical roughness of $90 \mathrm{~nm}$ rms. In both cases a top layer of $\mathrm{ZnO}$ is deposited with $13 \mathrm{~nm}$ and $90 \mathrm{~nm}$ rms roughness for the structure with smooth and corrugated interfaces, respectively. Fig. 1d summarizes the time domain characterization of the scattered radiation performed for these two nanostructures. For smooth internal interfaces the spectral amplitude of the scattered light (gray shaded area in inset Fig. 1d) is slightly narrower compared to the incident radiation (gray solid line). In the time domain the reconstructed scattered radiation field amplitude exhibits a single peak with $22 \mathrm{fs}$ duration (FWHM). The slight increase in comparison to the incident pulse duration is attributed to the narrower bandwidth of the scattered light.

a)

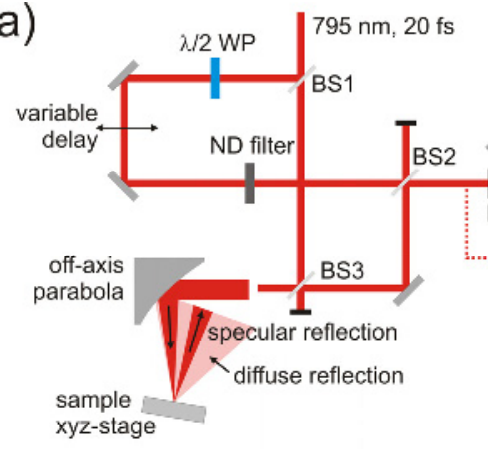

C)

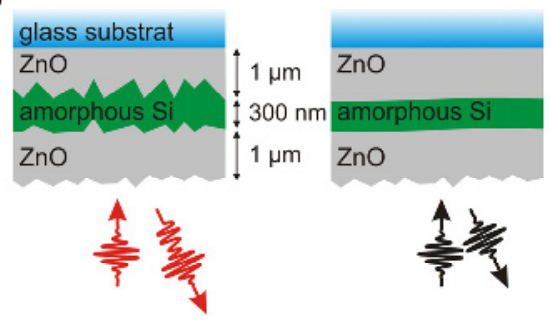

b)

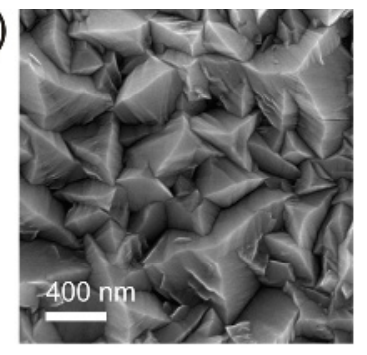

d)

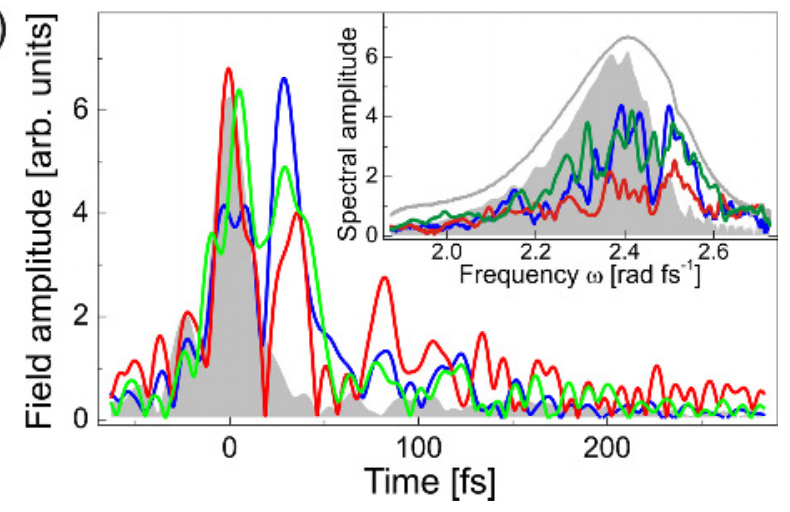

Fig. 1. Dual channel spectral interferometry of backscattered light from layered nanostructures. a) Scheme of the experimental setup. b) Scanning electron microscopy image of the ZnO layer in a nanostructured thin film silicon solar cell. c) Cross section through the layered nanostructures. The left structure corresponds to a thin film solar cell with omitted metallic back reflector. The structure with smooth internal interfaces $(<5 \mathrm{~nm} \mathrm{rms}$ roughness) serves as reference. d) Back-scattered field amplitudes from a layered structure with nano-textured internal interfaces (colored lines correspond to three different positions) and with smooth internal interfaces (shaded area). The corresponding spectral amplitudes are shown as inset together with the spectral amplitude of the laser (gray solid line). In all cases the polarization component of the scattered light parallel to the incident field is shown.

The sample with corrugated internal interfaces exhibits a completely different behavior. The scattered light shows a similar fast initial rise. However, in contrast to the sample with smooth internal interfaces it exhibits delayed scattered light components spread over more than $100 \mathrm{fs}$. The time dependent field amplitudes vary significantly for different positions on the sample. In addition, the spectral amplitude shows a highly structured response that also varies significantly for different 
positions on the sample and thus the strong modulation of the time-dependent field amplitude is attributed to beating between different modes. An average trapping lifetime of about $150 \mathrm{fs}$ is determined by fitting an exponential to the sum of the time-dependent field amplitudes recorded for 10 different focal spots on the sample (not shown). The resulting signal corresponds to the incoherent sum of the back-scattered light from the different locations. In a coherent sum of the scattered light - as it would be measured using a large focal spot for illumination averaging over many different light trapping modes - the lifetime effect cancels out because of destructive interference of the different spectral components. Hence, focused illumination and characterization of the coherent back-scattering provides additional information compared to usual light scattering measurements.

In contrast to the sample with smooth internal interfaces the nano-textured layered structure shows back-scattered light components that are polarized perpendicular to the incident pulse and exhibit similar effective lifetime as the non-rotated components. Assuming an effective refractive index of 3 for light propagation in the layered structure the lifetime of about 150-200 fs corresponds to an optical path of $15 \mu \mathrm{m}$. The observed spectral resonances indicate the formation of modes, i.e. closed multiple scattering pathways, and thus an upper limit for the lateral localization of the modes of about $5 \mu \mathrm{m}$ is estimated.

\section{Conclusion}

Dual channel spectral interferometry of back-scattered radiation allows investigating light trapping mechanisms in layered nanostructures. Coherence lifetimes of about $150 \mathrm{fs}$, mode beating, and resonances in the spectral amplitude indicate the formation of nanolocalized photonic modes that are attributed to the formation of closed pathways of multiply scattered radiation. The measurements show that microscopic spectral interferometry is a promising technique for the characterization and monitoring of thin film processing and provides a new approach to investigate and optimize light trapping mechanisms.

\section{References}

1. E. Yablonovitch and G. D. Cody, IEEE Trans. Elec. Devices 29, 300 (1982).

2. C. Haase and H. Stiebig, Appl. Phys. Lett. 91, 061116 (2007).

3. P. Obermeyer, C. Haase, and H. Stiebig, Appl. Phys. Lett. 92, 181102 (2008).

4. M. Agrawal and P. Peumans, Optics Express 16, 5385 (2008).

5. D. Domine, F. J. Haug, C. Battaglia, and C. Ballif, J. Appl. Phys. 107, 044504 (2010).

6. A. V. Shchegrov, A. A. Maradudin, and E. R. Mendez, in Progress in Optics, (Elsevier, Amsterdam, 2004), pp. 117-241.

7. D. S. Wiersma, P. Bartolini, A. Lagendijk, and R. Righini, Nature 390, 671 (1997). 